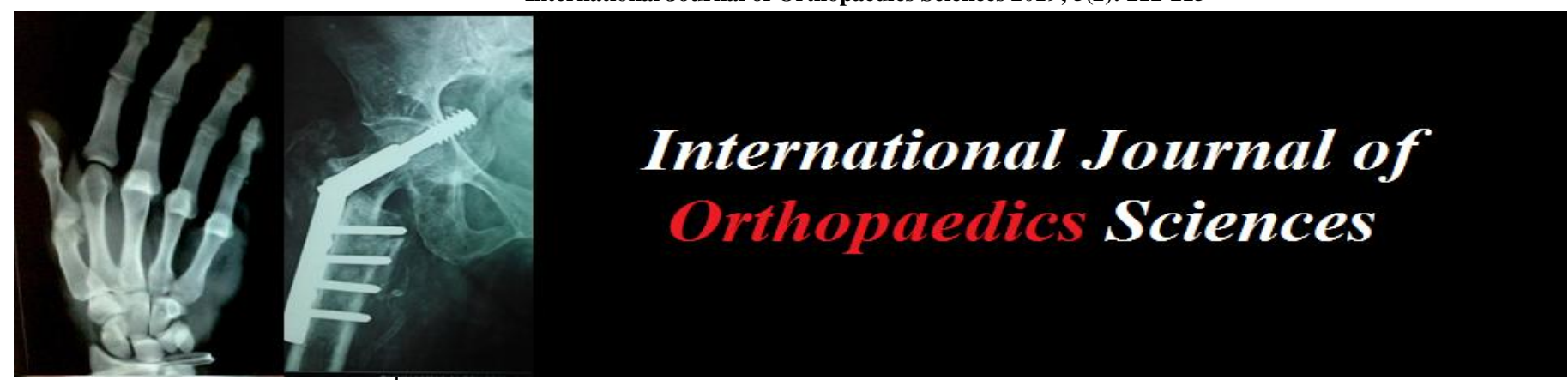

ISSN: $2395-1958$

IJOS 2019; 5(2): 212-215

(C) 2019 IJOS

www.orthopaper.com

Received: 01-02-2019

Accepted: 03-03-2019

Dr. Tushar Kanti Ghorai Ex-Junior Resident, DNB (ortho), Tata Main Hospital,

Jamshedpur, Jharkhand, India

Dr. Raghubaran Sharan

Ex-Senior Consultant of

Orthopaedics, MS (Ortho), Tata

Main Hospital, Jamshedpur,

Jharkhand, India
Correspondence

Dr. Tushar Kanti Ghorai

Ex-Junior Resident, DNB

(ortho), Tata Main Hospital,

Jamshedpur, Jharkhand, India

\section{A prospective study on functional outcome in high tibial medial open wedge osteotomy in medial compartmental osteoarthritis of knee}

\author{
Dr. Tushar Kanti Ghorai and Dr. Raghubaran Sharan
}

DOI: https://doi.org/10.22271/ortho.2019.v5.i2e.21

Abstract

Osteoarthritis is a degenerative joint disease result from breakdown of joint cartilase and underlying bone. In medial unicompartmental osteoarthritis of knee with varus deformity, a valgus realignment high tibial osteotomy (HTO) is a treatment option in selective active patients. In this study, we evaluated functional outcome in medial open wedge high tibial osteotomy in selected patients.

This study was conducted with twenty young active patients with medial compartmental osteoarthritis allocated for medial biplanar open wedge high tibial osteotomy, stabilised with non-locking plate with or without autologous bone grafting or bone graft substitute. All patient assessed pre-operative and postoperative period by Knee society score, VAS score.

In our study, range of movement, Knee score, pain score, functional score, over all final result according to knee society score improved that is statistically significant.

Keywords: HTO High tibial osteotomy, KSS knee society score, VAS visual analogue scale

\section{Introduction}

Knee osteoarthritis is one of most common disorder with aging ${ }^{1}$ and cause considerable pain, immobility, reduction of activity, malalignment mainly in elderly and obese people ${ }^{[2,3]}$. Now a days, its incidence has increased in significant number of comparatively younger population. In medial unicompartmental osteoarthritis of knee with varus alignment $[4,5]$, a valgus realignment ${ }^{[6]}$ high tibial osteotomy is a treatment option in selective active patients ${ }^{[7]}$. Principle ${ }^{[8]}$ of high tibial osteotomy is to redistribute ${ }^{[9-12]}$ weight bearing laod from arthritic portion to viable articular cartilage portion of knee ${ }^{[12]}$. It becomes a mandatory operation adjunct to articular cartilage restoration when there is preoperative malalignment [26]. Advantage of high tibial osteotomy includes preservation of joint and induction of biological remodelling with reduced synovitis, local osteosclerosis and repair of articular cartilage [20-25], delay the need for an arthroplasty ${ }^{[27]}$.

A high tibial medial open wedge osteotomy ${ }^{[26,27]}$ has proved to be effective in mild to moderate osteoarthritis of medial compartment of knee in active young individuals, is less invasive, safe, easier to perform, correction of deformity close to origin, provides more predictable correction intraoperatively, better preservation of bone stock, avoid peroneal nerve injury and proximal tibio-fibular joints disruption [30], avoid compartment syndrome ${ }^{[31]}$, less expansive and the relative ease of combining it with other procedures. It reduces pain, improves mobility, range of movement, activity, survival ${ }^{[32-34]}$.

In this study medial biplanar open wedge high tibial osteotomy was stabilised with nonlocking plate with or without autologous bone grafting or bone graft substitute.

This study, "a prospective study on functional outcome in high tibial medial open wedge osteotomy in medial compartmental osteoarthritis of knee" has been taken to evaluate functional outcome in medial open wedge high tibial osteotomy in selected patients.

\section{Aims}

Aims of study is to evaluate functional outcome of high tibial medial wedge osteotomy in medial compartment osteoarthritis of knee. 
Objectives of study is to assess functional outcome in high tibial medial wedge osteotomy in unicompartmental osteoarthritis of knee in terms pain relief, range of movement (ROM), correction of deformity, functional outcome using knee society scoring system.

\section{Patients (Materials) and Methods}

The study is a prospective and observational study conducted in the department of Orthopedics, Tata Main Hospital, Jamshedpur, between November 2015 to October 2016 including the patients admitted at orthopedics department with knee pain and varus deformity. Approval by the ethics committee of Hospital, Jamshedpur had taken and written informed consent of patients obtained to conduct the study. Total study population was 25 out of that we are considering only $80 \%$ of that population. Taking the $\alpha$ at 0.05 and desired power of study is $80 \%$ the sample size needed is 20 .

\section{Inclusion criteria}

1. Pain and disability resulting from osteoarthritis in active patient

2. Age: $<60$ years

3. Evidence of medial compartment involvement

4. Patient must able to use crutches / walker

5. Patient should have sufficient muscle strength and motivation to carry out rehabilitation program

6. $\mathrm{BMI}<35$

\section{Exclusion criteria}

1. Bi-compartment or tri-compartment involvement

2. Lateral subluxation of tibia $>1 \mathrm{~cm}$

3. Flexion contracture $>200$

4. Knee flexion $<900$

5. Medial compartment tibia bone loss $>3 \mathrm{~mm}$

6. $>20^{\circ}$ of varus correction is needed.

7. Present or past history of inflammatory arthritis

8. Significant peripheral vascular disease

9. $\quad$ BMI $>35$

\section{Methodology}

All patients admitted for medial compartment osteoarthritis with varus deformity of knee in orthopedics department in Tata Main Hospital, Jamshedpur, Jharkhand from 5th November 2015 to 4th October 2016, were examined clinically, radiologically and patient will be taken for high tibial medial open wedge osteotomy according to inclusion and exclusion.

Patients were operated with open wedge high tibial osteotomy, fixation was done by non-locking T-buttress plate, augmentation done by autologous bone graft or synthetic bone graft according to opening gap (>13mm).

Clinically patients was assessed according to Knee society scoring system, range of knee movement by goniometer and radiologically X-ray was taken from hip to ankle by PACS in DICOM system. Ahlbäck radiohraphic grade used for grading of osteoarthritis. Also pain was assessed in VAS score.

Pre-operatively desire correction angle deformity measured by Miniac technique, per-operative mechanical axis had been checked by cable method.

Pre-operative and postoperative evaluation of medial compartment osteoarthritis by Ahlbäck score, various malalignment by aTFA (anatomical tibio-femoral axis), MPTA (Mechanical proximal tibial angle), JLCA (joint line convergence angle), mTFA (mechanical tibio-femoral angle), PTS (posterior tibial slope), severity of pain measured on visual analog scale (VAS), knee function according to Knee Society Score ${ }^{[26]}$ are to be measured.

Patients will be follow up clinically, radiologically at 6week, 3rd, 6th month of postoperative period. Grading of results done according to final KSS score.

BMI, early and late complication and additional surgery such as removal of implant or other complication would be noted.

\section{Statistical Method}

The collected data organized, tabulated and statistically analysis using "MedCalc". The data will be analyzed by appropriate statistical tools.

\section{Results}

Present study was conducted at the department of orthopaedics, Tata Main Hospital, Bistupur, Jamshedpur, Jharkand from November 2015 to October 2016 on 20 comparatively young patients of medial compartmental osteoarthritis of knee. This study was conducted with aims and objectives of improving clinical and radiological, functional outcome. In our study mean age of the patients was 50 year $(50.75 \pm 5.63)$. Out of 20 patients, 14 patients are female and rest 6 patients are male. Nearly $45 \%$ women over the age of 65 year have symptoms while radiological evidence is found in $70 \%$ of those over 65years. OA is a major cause of mobility impairment, particularly among female ${ }^{[26]}$. Out of 20 knees, $75 \%$ were right and $25 \%$ were left. All patients were right dominant. Osteoarthritis of knee in young active age group is more common in dominant side.

Mean height of all patients was 1.63 meter. Post-operatively there was no limb length discrepancy. Mean weight of patient was $65.25 \mathrm{~kg}$, mean BMI was 24.06. Mean knee pain score was $17 \pm 6.57$ pre-operatively, in post- operative period was $43 \pm 5.23$.

Mean pre-operative range of movement (ROM) was $115.25 \pm$ 8.96. Post-operative ROM improved in all patients except one who has lateral condyle of tibia fracture intra-operatively. None of our patients developed extension lag. Mean postoperative ROM is $124 \pm 4.47$.

In 20 patients no one has preoperative antero-posterior instability. Only one patients had $6-9 \mathrm{~mm}$ medio-lateral instability. There was no change of stability in any patients after surgery.

In all patients, knee society score (KSS part I) improved significantly. Preoperative mean knee score was $41.95 \pm 8.39$, postoperative knee score was $89.35 \pm 9.31$.

Mean preoperative functional knee score was $46.5 \pm 9.05$, which improved in all patients postoperatively, postoperative mean functional knee score was 79.25 \pm 5.68 .

Preoperative Ahlbäck radiohraphic grade was grade II for 11 patients $(55 \%)$, grade III was for 9 patients $(45 \%)$. Within our followup period Ahlbäck grading was unchanged. Preoperative anatomical femero-tibial angle (aFTA) was $2.950 \pm 0.76$ varus, and postoperative aFTA was $6.750 \pm 0.87$ valgus.

In our study, pre-operative mTFA was $8.8 \pm 1.58$ (varus) and post-operative mTFA was $3.05 \pm 0.76$, mean change 11.850 .

Mean preoperative joint line convergence angle (JLCA) was $3.680 \pm 0.75$. After HTO, it had been reduced in all patients. Mean postoperative JLCA was $1.850 \pm 0.37$. Mean chage of JLCA is 1.230 .

Preoperative mean mechanical medial proximal tibial angle (MPTA) was $84 \pm 1.34$. Postoperative mean MPTA was 92.85 \pm 1.75 .

Preoperative mean axis deviation (MAD) of all patients was 
$19.43 \pm 2.09 \mathrm{~mm}$ (varus), after HTO postoperative mean MAD was $11.78 \pm 1.18$ valgus.

Posterior Tibial Slope (Moore -Heavy method) pre-operative was $7.7 \pm 0.66$, post-operative was $10.95 \pm 0.81$, mean increased was $3.25^{\circ}$.

There was no non-union in any osteotomy site in this present study. In $90 \%$ cases, osteotomy united withih 12 week. We had applied iliac crest bone graft in case of osteotomy $>13^{\circ}$.
We had applied bone graft in 6 patients, autologous bone graft in five patients and synthetic bone graft one patients,

Regarding complication, two patients had superficial wound infection which was healed by regular dressing and changing of antibiotics. There was lateral condyle of tibia fracture in one patient during operation. Finally there is significant improvement of range of knee movement, reduction of pain, improvement of KSS score.

Table 1: Comparison of different scoring among the patients during the given time period.

\begin{tabular}{|c|c|c|c|c|}
\hline \multirow{2}{*}{ Parameters } & ROM & Knee scores & Pain scores & Functional scores \\
\cline { 2 - 5 } & Mean \pm s.d & Mean \pm s.d & Mean \pm s.d & Mean \pm s.d \\
\hline 6 weeks & $117.25 \pm 7.34$ & $81.95 \pm 9.74$ & $28.5 \pm 5.87$ & $51.25 \pm 18.42$ \\
\hline 3 months & $120.75 \pm 4.37$ & $86.65 \pm 9.34$ & $37.75 \pm 4.72$ & $66.75 \pm 8.32$ \\
\hline 6 months & $123.5 \pm 5.15$ & $89.35 \pm 9.31$ & $43 \pm 5.23$ & $79.25 \pm 5.68$ \\
\hline $\mathrm{F}_{\text {cal }}$ & 38.43 & 3.13 & 5.91 & 26.79 \\
\hline $\mathrm{P}-$ value & $\mathrm{P}<0.05$ & $\mathrm{P}<0.05$ & $\mathrm{P}<0.05$ & $\mathrm{P}<0.05$ \\
\hline Conclusion & significant & significant & significant & significant \\
\hline
\end{tabular}

Table 2: Final result according to knee society score as followed.

\begin{tabular}{|c|c|c|}
\hline Final results & No. of patients & Percentage \\
\hline Excellent & 16 & $80 \%$ \\
\hline Good & 3 & $15 \%$ \\
\hline Fair & 1 & $5 \%$ \\
\hline Poor & 0 & $0 \%$ \\
\hline
\end{tabular}

We had taken the patients in early osteoarthritis in relatively active patients, no mediolatearl or anterioposterio instability, no flexion deformity, stable fixation done, bone graft was applied with gap > 13mm intra-operatively, early range of movement started, results was excellent in $80 \%$ cases.

Common problems with high tibial osteotomy is difficulty in achieving an accurate degree of correction. The ideal angular correction for the best long-term outcome is unclear. Poor results have been reported where undercorrection of the deformity has occurred. Even when a specific angle of correction has been planned pre-operatively, it has been reported that optimal correction is not achieved in $20 \%$ of HTOs. This may be due to difficulty in standardising the radiographs, rotational malalignment may affect the perceived deformity. It has also been shown that there is no correlation between knee alignment and pain and therefore static correction of the deformity alone may not be reliable.

Slight overcorrection in HTO produces more satisfying results. However, the optimal degree of valgus angulation is still controversial. Dugdale et al. recommended $3^{0}-5^{0}$ valgus mechanical axis, whereas other authors suggested $3^{0}-6^{0}, 3-7^{0}$, or $7-9^{0}$. Conventry et al. reported that the 10-year survival rates were $63 \%$ in knees with 50 valgus angulation, $87 \%$ in knees with $60-70$ valgus angulation, and $94 \%$ in knees with $\geq 80$ valgus angulation.

\section{Conclusion}

In this present study, our procedure of high tibial medial open wedge osteotomy is very effective in active young patients with uni-compartmental osteoarthritis of knee. We had fixed the osteotomy gap with T-buttress plate. This procedure can offer freedom from pain, excellent range of movement and functional outcome with minimal post-operative complications. This osteotomy is comparatively easy to perform than lateral closing wedge osteotomy. With the calibrated osteotome guide, we achieved précised osteotomy angle which is very important for achieving post-operatively satisfactory results. It is a bone preserving surgery. It is usually a time buying procedure to delay eventual total knee arthroplasty.

This is a deformity correcting surgery so it has a steep learning curve.

Though the long term results deteriorate with time but are still gratifying enough for the Indian patient as well as for those of the third world, who cannot afford the costlier surgeries and implants and also are reluctant to change their squatting habits and job profile. In our society, people used to try bear pain till the situation goes beyond control. So getting young osteoarthritis patients with proper indication is not easy and convincing them about this procedure was another deal.

\section{Acknowledgements}

Special thank to Dr. Santosh Rawat, HOD, department of orthopaedics, Tata Main Hospital, Jamshedpur who encourage me to do this work.

\section{References}

1. Kellgren JH, Lawrence JS. Radiological assessment of osteoarthrosis. Ann Rheum Dis. 1957; 16:494-502.

2. Lawrence JS, Bremner JM, Bier F. Osteo-arthrosis. Prevalence in the population and relationship between symptoms and x-ray changes. Ann Rheum Dis. 1966; 25:1-24.

3. Peyron JG. Osteoarthritis. The epidemiologic viewpoint. Clin Orthop Relat Research. 1986; 213:13-9.

4. Chaturong Pornrattanamaneewong, Surin Numkanisorn, Keerati Chareancholvanich, Thossart Harnroongroj. A retrospective analysis of medial opening wedge high high tibial osteotomy for varus osteoarthritic knee. Indian J Orthop. 2012; 46:455-61.

5. Kongcharoensombat W. Clinical Outcome of Medial Opening Wedge Osteotomy with T-Locking Plate: Two Years Follow-Up. Malaysian Orthopaedic. 2014; 8:50-6.

6. Jackson JP, Waugh W, Green JP. High tibial osteotomy for osteoarthritis of the knee. J Bone Joint Surg Br. 1969; 51:88-94.

7. Han JH, Kim HJ, Song JG, Yang JH, Bhandare NN, Fernandez AR et al. Is Bone Grafting Necessary in Opening Wedge High Tibial Osteotomy? A MetaAnalysis of Radiological Outcomes. Knee Surg Relat Res. 2015; 27(4):207-20. doi: 10.5792/ksrr.2015.27.4.207.

8. Maquet P. The biomechanics of the knee and surgical possibilities of healing osteoarthritic knee joints. Clin Orthop Relat Res. 1980; 146:102-10. 
9. Terauchi M, Shirakura K, Kobuna Y, Fukasawa N. Axial parameters affecting lower limb alignment after high tibial osteotomy. Clin Orthop Relat Res. 1995; 317:1419.

10. Huang TL, Tseng KF, Chen WM, Lin RM, Wu JJ, Chen TH. Preoperative tibiofemoral angle predicts survival of proximal tibia osteotomy. Clin Orthop Relat Res. 2005; 432:188-95.

11. Thami Benzakour, Abderrazak Hefti, Mohamed Lemseffer, Jamal Dine El Ahmadi, Houssam Bouyarmane, Ahmed Benzakour. High tibial osteotomy for medial osteoarthritis of the knee: 15 years follow-up International Orthopaedics (SICOT). 2010; 34:209-15.

12. Frankel VH, Burstein AH, Brooks DB. Biomechanics of internal derangement of the knee. Pathomechanics as determined by analysis of the instant centers of motion. J Bone Joint Surg Am. 1971; 53:945-62.

13. Pal CP, Singh P, Chaturvedi S, Pruthi KK, Vij A. Epidemiology of knee osteoarthritis in India and related factors. Indian J Orthop. 2016; 50(5):518-22.

14. Mankin HJ. The reaction of articular cartilage to injury and osteoarthritis (first of two parts). N Engl J Med. 1974; 291:1285-92.

15. Johnson RJ, Kettelkamp DB, Clark W, Leaverton P. Factors effecting late results after meniscectomy. J Bone Joint Surg Am. 1974; 56:719-29.

16. Muir H. Heberden Oration, Molecular approach to the understanding of osteoarthrosis. Ann Rheum Dis. 19761977; 36:199-208.

17. Hamerman D, Klagsbrun M. Osteoarthritis. Emerging evidence for cell interactions in the breakdown and remodeling of cartilage. Am J Med. 1985; 78:495-9.

18. Maquet P. Valgus osteotomy for osteoarthritis of the knee. Clin Orthop Relat Res. 1976; 120:143-8.

19. Ivarsson I, Myrnerts R, Gillquist J. High tibial osteotomy for medial osteoarthritis of the knee. A 5 to 7 and 11 year follow-up. Bone \& Joint Journal. 1990; 72-B(2):238-44.

20. William M, Mihalko. Arthroplasty of the knee. In: Frederick M Azar, S. Terry Canale, James H. Beaty. Campbell's Operative Orthopaedics, 13th Edition. Memphis: Elsevier, 2016, 443.

21. Agarwala S, Sobti A, Agrawal P. A report of nonunion at medial wedge high tibial osteotomy site and its management. J Nat Sci Biol Med. 2015; 6(1):S160-2. doi: 10.4103/0976-9668.166128.

22. Akamatsu Y, Koshino T, Saito T, Wada J. Changes in osteosclerosis of the osteoarthritic knee after high tibial osteotomy. Clin Orthop Relat Res. 1997; 334:207-14.

23. Sprenger TR, Doerzbacher JF. Tibial osteotomy for the treatment of varus gonarthrosis. Survival and failure analysis to twenty-two years. J Bone Joint Surg Am. 2003; 85:469-74.

24. Devgan A, Marya KM, Kundu ZS, Sangwan SS, Siwach RC. Medial Opening Wedge High Tibial Osteotomy for osteoarthritis of Knee: Long Term Results in 50 Knees. Med J Malaysia. 2003; 58:62-8.

25. Nelissen EM, Van Langelaan EJ, Nelissen RG. Stability of medial opening wedge high tibial osteotomy: a failure analysis. Int Orthop. 2010; 34(2):217-23. doi: 10.1007/s00264-009-0723-3.

26. Insall JN, Dorr LD, Scott RD, Scott WN. Rationale of the Knee Society clinical rating system. Clin Orthop Relat Res. 1989; (248):13-4. 